\title{
Automatic Targeting Method and Accuracy Study in Robot Assisted Needle Procedures
}

\author{
Alexandru Patriciu, Dumitru Mazilu, Doru Petrisor, Louis Kavoussi, and \\ Dan Stoianovici \\ URobotics Laboratory \\ Johns Hopkins Medical Institutions \\ 5200 Eastern Ave., Baltimore, MD \\ http://urology.jhu.edu/urobotics
}

\begin{abstract}
We present an automatic needle targeting method for fluoroscopyguided percutaneous access procedures. The approach is derived from the manual needle superimposition technique making it intuitive and familiar to surgeons and radiologists. The proposed algorithm is insensitive to image distortion and does not require any C-Arm calibration or initial pose estimation. Needle alignment is performed using a direct adaptive visual servoing approach; once the desired orientation is achieved, insertion is performed using under joystick control. The algorithm was implemented and tested using our AcuBot robot, purposely built for percutaneous image guided interventions. A series of tests were performed showing that the proposed approach increases accuracy and reduces radiation exposure.
\end{abstract}

\section{Introduction}

Minimally invasive procedures are frequently used for the treatment of different medical conditions due to their potential of improving the recovery time and reducing patient trauma. Such procedures require the insertion of instruments with high accuracy at specific targets inside the body. Usually, the surgeon uses x-ray images for the target identification and instrument guidance. The most common imager available in the operating room is a mobile fluoroscopic, a C-Arm. These may however deliver cumulative amounts of radiation to the patient and surgeon. A solution for reducing the radiation exposure and simultaneously increasing accuracy was offered by the development of robot manipulators adapted to surgical tasks. An overview of several surgical robotic systems and clinical applications is presented in [1]. A common kinematic feature in many surgical robots is the ability of orienting a surgical instrument about a pivot point conveniently located on the instrument itself. This feature is named Remote Center of Motion (RCM) and in percutaneous procedures allows for orienting the needle while maintaining the location of its point at the desired skin entry point.

Several authors explored the needle placement task using C-Arm imagers and two main directions were approached. The first is to compute the target position in robot space, when one needs to register the robot space with respect to the C-Arm space and identify the target in the imager space using two dissimilar C-Arm orientations. Then, 
the position of the target is readily transformed from the C-Arm to the robot space. Several registration methods have been proposed over the years. For example, Taylor et al. [2, 3] developed a registration method using a corkscrew fiducial object attached to the robot end-effector. An algorithm was developed to find the 6DOF transformation from the robot to the C-Arm. This class of algorithms requires image distortion correction using additional hardware mounted on the C-Arm detector. While these methods provide good accuracy, the additional hardware required for image dewarping could impede target identification.

A second approach is to use properties of the perspective projection and perform the targeting task without explicitly computing the position of the target. Navab and Loser explored this approach [4]; their method uses visual servoing in two different views in order to achieve proper instrument orientation. The instrument insertion depth is computed using cross-ratio invariants. Our group also developed a similar targeting method [5] moving the needle on a conical trajectory in one C-Arm orientation and using visual servoing a second C-Arm orientation. Both of these methods assume that the $\mathrm{C}$-Arm projects 3D lines in 2D lines, which is reasonable as long as the region of interest is close to the center of the image.

The method reported here is also based on visual servoing and relaxes the nondistortion assumption. This algorithm is theoretically capable of achieving zero alignment error. The method was implemented and tested using our AcuBot robot [6].

\section{Method}

The approach automates the needle superimposition technique manually practiced by the surgeon by using adaptive visual servoing. The needle alignment requires one single C-Arm orientation. A lateral view is then used for needle insertion. In the first C-Arm position the needle point is placed at the skin entry point which is superimposed over the target. The needle is then rotated about that point until its head overlaps it in the image, like in the manual case. This guarantees that the target is on the needle path. The needle is then inserted under a lateral C-Arm view.

In the initial orientation the X-ray source, the target and the needle tip are collinear defining the line $\delta$ (Fig. 1). The problem at hand is to align the needle along this direction. In image space, the alignment position is identified by the superposition of the needle head over its point. In control terms this is a set-point regulation task using a non-calibrated camera with fixed but unknown position and orientation.

\subsection{Control Algorithm}

The visual servoing problem has been intensively addressed in industrial robotics since early 80's as a modality to improve precision in positioning tasks. In general, visual-servoing uses visual information to control the pose of a robot end-effector [7]. For instance, a standard architecture uses a fixed camera(s) to track the robot endeffector towards a desired position. Initially, visual-servoing controllers were developed for cameras with known optic parameters and position. Recently, Hespanha et al. showed what tasks can be performed with an un-calibrated camera[8] and adaptive 
control methods have been proposed for the un-calibrated visual-servoing of planar manipulators [9, 10]. The proposed algorithm is an image-based direct visual servoing using a fixed un-calibrated X-Ray camera. Compared to the industrial case, in our surgical case the marker (needle head) moves on a sphere rather than a plane. However, since the initial orientation of the needle is closed to the desired orientation, the industrial approach can be modified to serve the new purpose.

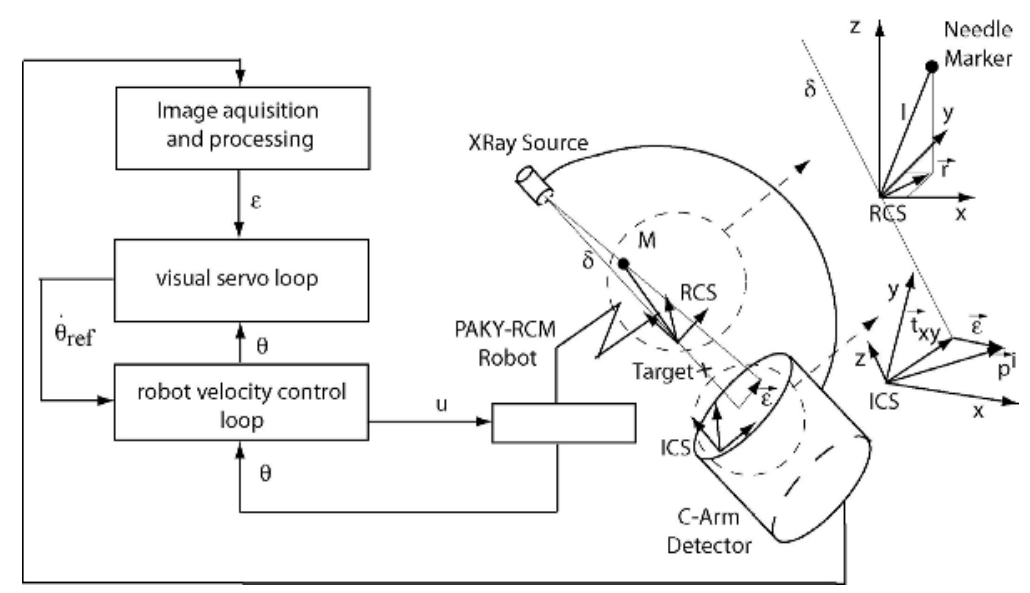

Fig. 1. Needle targeting diagram

Control equations may thus be constructed in a similar manner, but due to the particular trajectory of the marker, the resulting error equations contain nonlinear terms of joint coordinates, as follows.

The RCS coordinate system (Fig. 1) is conveniently centered at the point of the needle and aligned with its $\vec{z}$ axis along the initial direction of the needle. The marker position in RCS then given by:

$$
\vec{p}^{r}=\left(\begin{array}{lll}
r_{1} & r_{2} & \sqrt{l^{2}-r_{1}^{2}-r_{2}^{2}}
\end{array}\right)^{T}
$$

where, $l$ is the length of the needle and $\vec{r}=\left(\begin{array}{ll}r_{1} & r_{2}\end{array}\right)^{T}$ is the position of the needle head in the $x O y$ plane of RCS. The unknown transformation from the RCS to the image coordinate system (ICS) can be represented by a sequence of rotations $R_{z}(\alpha) R_{y}(\beta) R_{x}(\gamma)$ followed by a translation $\vec{t}=\left(\begin{array}{lll}t_{x} & t_{y} & t_{z}\end{array}\right)^{T}$, where $\vec{t}$ defines the position of the needle tip in ICS. Thus, the marker position in ICS is:

$$
\vec{p}^{c}=R_{z}(\alpha) R_{y}(\beta) R_{x}(\gamma) \vec{p}^{r}+\vec{t}
$$

Consider $\vec{p}^{i}$ the perspective projection of $\vec{p}^{c}$ on the detector plane and $\vec{t}_{x y}$ the projection of the needle tip on the same plane. The needle is aligned with the target if $\vec{p}^{i}=\vec{t}_{x y}$, thus the alignment error can be defined as: 


$$
\varepsilon=\vec{p}^{i}-\vec{t}_{x y}
$$

Taking the time derivative of (3), the error equation becomes:

$$
\dot{\varepsilon}=\widetilde{R}_{z}(\alpha) M(\vec{r}, \beta, \gamma) \dot{\vec{r}} ; \tilde{R}_{z}(\alpha)=\left(\begin{array}{cc}
\cos (\alpha) & -\sin (\alpha) \\
\sin (\alpha) & \cos (\alpha)
\end{array}\right)
$$

and $M(\vec{r}, \beta, \gamma)$ a known nonlinear matrix function of known (current needle position) and unknown parameters (initial position of the needle tip, initial needle orientation). The needle alignment requirements are met when $\varepsilon=0$. A feedback controller is used to stabilize the system (Eq. 4) in the origin for appropriate initial conditions. Assuming that the implementation uses a fast inner loop for the robot velocity control, we can concentrate on the kinematic problem and generate robot reference velocities $\dot{\theta}$ such that the error $\varepsilon$ approaches 0 .

Since the robot is initially close to the desired position it can be proved that the matrix $M$ is bounded for all robot positions and the rotation about the $\vec{z}$ axis induced by $M$ is small compared to $\alpha$. A stabilizing control law can then be chosen as:

$$
\dot{\vec{r}}=-K_{p} \widetilde{R}_{z}(\hat{\alpha})^{T} \varepsilon ; K_{p}=K_{p}^{T}>0 \in R^{2} ; \hat{\alpha} \text { an estimated value for } \alpha .
$$

Then, the robot reference velocities can be computed as:

$$
\dot{\vec{\theta}}=J(\vec{\theta})^{-1} \dot{\vec{r}}
$$

where, $\vec{\theta}$ are the RCM joint coordinates, and $J(\vec{\theta})$ is the Jacobian of the $\vec{\theta}$ to $\vec{r}$ transformation.

Since the visual control loop runs at low frequency (approximately 30Hz), a discrete method was designed for the estimation of $\alpha$ :

$$
\hat{\alpha}_{k}=\hat{\alpha}_{k-1}+\angle\left(\varepsilon_{k}-\varepsilon_{k-1},-\varepsilon_{k-1}\right) \text { if } \varepsilon_{k-1} \neq 0 \text { and } \hat{\alpha}_{k}=\hat{\alpha}_{k-1} \text { otherwise; }
$$

where, $\hat{\alpha}_{k}, \varepsilon_{k}$ estimated $\alpha$ respectively error at step $\mathrm{k}$ and $\angle$ computes the angle between the two parameter vectors.

Equations (5), (6) and (7) provide a feedback control law that stabilizes (4) in the origin which aligns the needle towards the target. The method was successfully implemented using the AcuBot robot.

\subsection{The AcuBot}

AcuBot comprises two robotic manipulators connected by a 6DOF passive arm. The base manipulator is a Cartesian stage attached to the operating table through an adapter. The second manipulator is the PAKY-RCM [11] module. The passive arm and the Cartesian stage allow for the initial positioning of the robot such that the tip of the needle is at the skin entry point. The RCM provides the 2 rotational DOF required for needle orientation; PAKY is a needle driver used once proper orientation is achieved. 
The manipulator is controlled using an industrial PC computer equipped with a motion control card "Motion Engineering Inc" PCX-DSP, and a frame grabber Matrox Meteor II. The motion control card is used as a velocity controller for the RCM robot and receives reference joint velocities from the "visual servo loop". The visual servo loop implements the equations (5), (6) and (7). The Matrox Meteor II board and Matrox Imaging Library are used for image acquisition and processing. In order to easily recognize the end of the needle in the image, a marker represented by a $5 \mathrm{~mm}$ steel ball is attached to the needle. The control was developed and initially tested using a CCD camera instead of the C-Arm for reducing radiation exposure.

\section{Results}

A first set of experiments was conducted to test and prove targeting accuracy. A comparative study was then designed for assessing the feasibility and usefulness of the proposed method. A percutaneous procedure (kidney access) was simulated in two different settings. In the first, automatic alignment was used for needle orientation whereas in the second the robot was manually driven using a joystick control. Both the accuracy of needle placement and the alignment time were recorded and used in the evaluation.

\subsection{Automatic Targeting Tests}

The algorithm was implemented and initially tested with a CCD camera in place of the C-Arm. The maximum needle placement error in 50 experiments, measured as the distance between the point of the needle and the $1 \mathrm{~mm}$ ball target was $1 \mathrm{~mm}$. The convergence of the visual servoing algorithm was also tested for various camera positions and initial orientations of the needle. Figure 2 shows 6 examples of the algorithm's convergence by displaying the trajectory of the needle head towards overlapping the point of the needle in image coordinates.

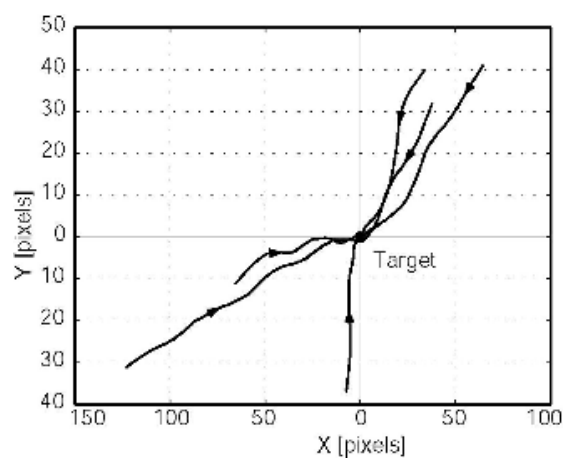

Fig. 2. Visual Servoing Convergence Graph 
A second set of experiments was performed with the C-Arm. AcuBot was attached to an OR table and an OEC $9800 \mathrm{C}$-Arm was used as imaging device. Several targets defined by $1 \mathrm{~mm}$ steel balls were placed in different calyces of a kidney phantom (Percutaneous Kidney Slab, Limbs and Things Inc). The model was manufactured from silicone rubber with mechanical characteristics similar to the soft tissue.

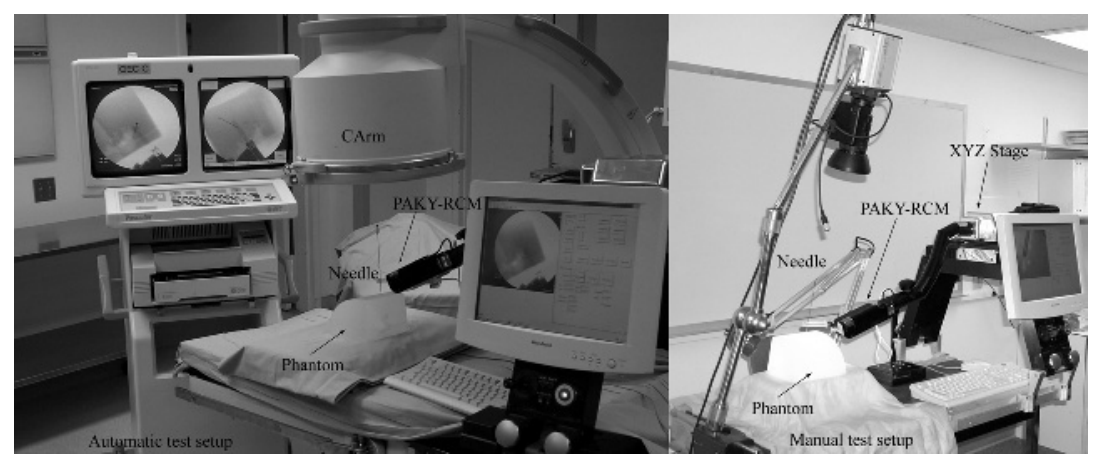

Fig. 3. Automatic needle alignment tests under x-ray fluoroscopy (left) and video camera guidance (right)

Fig. 3 shows two photographs of the experiments performed with the x-ray and video guidance. In both cases, a trial involved the following steps:

1. Define the target and load the needle in the PAKY driver.

2. Position the C-Arm and choose the entry point; drive the robot using joystick control until the needle tip is at the desired entry point (overlapped over the target).

3. Automatically align the needle towards the target using the proposed algorithm and measure the time.

4. Rotate the C-Arm in a lateral position and insert the needle under joystick control.

5. Estimate the precision of needle placement where error was defined as the distance between the needle tip and the surface of the target ball.

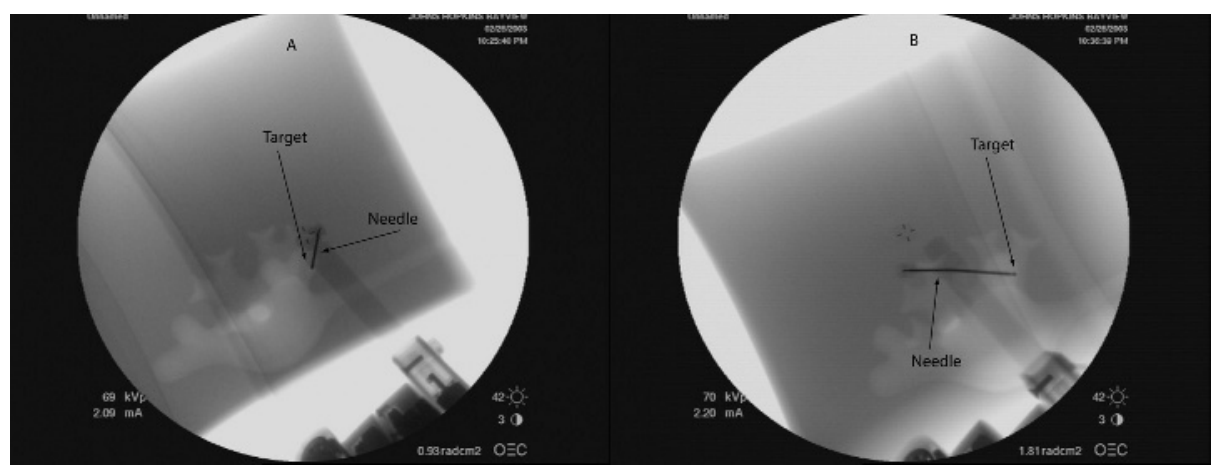

Fig. 4. Needle deployed at $1 \mathrm{~mm}$ ball target within kidney model 
The results of a series of 25 trials performed under $\mathrm{x}$-ray guidance are presented in Table 1 and a typical $x$-ray showing the needle targeting the steel ball is presented in Fig. 4.

Table 1. Statistic results of the automatic and joystick guided needle insertion

\begin{tabular}{|l|c|c|c|c|}
\hline \multirow{2}{*}{} & \multicolumn{2}{|c|}{$\begin{array}{c}\text { Automatic } \\
1 \mathrm{~mm} \text { target, 18G needle } \\
25 \text { experiments }\end{array}$} & \multicolumn{2}{c|}{$\begin{array}{c}\text { Joystick } \\
\text { 3mm target, 18G needle } \\
\text { 40 experiments }\end{array}$} \\
\cline { 2 - 5 } & Mean & Std. Dev. & Mean & Std. Dev. \\
\hline $\begin{array}{l}\text { Alignment } \\
\text { time }\end{array}$ & $5.54 \mathrm{~s}$ & $1.67 \mathrm{~s}$ & $10.56 \mathrm{~s}$ & $3.95 \mathrm{~s}$ \\
\hline Accuracy & $0.8 \mathrm{~mm}$ & $0.78 \mathrm{~mm}$ & $1.03 \mathrm{~mm}$ & $0.42 \mathrm{~mm}$ \\
\hline
\end{tabular}

\subsection{Manual Targeting Tests}

Forty trials were performed for the manual tests using the same type of kidney model. For minimizing the radiation exposure a CCD camera was used instead of the C-Arm. This was possible since the phantom used was made of translucent silicone rubber. In this case the targets used were $3 \mathrm{~mm}$ balls, because the $1 \mathrm{~mm}$ used in x-ray were hard to see with the camera. The balls were inserted in calyces of the kidney model. The steps of the trial described for the automatic alignment were also followed in this case; except for step \#3 were the subjects used the joystick control to align the needle towards the target. For each trial, alignment time and precision were recorded as summarized in Table 1.

These results indicate that even though the target was 3 times smaller the imageguided automatic procedure is twice times faster and more accurate than the joystick approach. This study is the first step in evaluating the performance of the proposed automatic alignment method. Future research involving animal experiments, will be needed to evaluate the influence of needle deflection and respiratory motion in targeting accuracy.

\section{Conclusion}

The paper presents a method for automatic needle placement under C-Arm fluoroscopy. The proposed algorithm uses a non-calibrated mobile C-Arm, widely available in the operating rooms, and a minimal marker represented by a steel ball attached to the head of the needle. The approach is intuitive and familiar to the surgeons being derived from the manual approach. The method was implemented using a robot purposely designed for image-guided needle procedures. A comparative study of the automatic versus joystick control of the robot showed an improvement in the procedure time and the accuracy.

Acknowledgement and Disclaimer. This work was partially supported by grant No. 1R21CA088232-01A1 from the National Cancer Institute (NCI). Its contents are 
solely the responsibility of the author and do not necessarily represent the official views of NCI.

Under licensing agreements between ImageGuide (iG) and the Johns Hopkins University (JHU), the authors are entitled to a share of royalty received by JHU on iG sales of the AcuBot robot described in this article. Under a private license agreement between D.S., L.R.K and $\mathrm{iG}, \mathrm{DS}$ and L.R.K are entitled to royalties on $\mathrm{iG}$ sales of products embodying the AcuBot technology. The authors and JHU own iG stock, which is subject to certain restrictions under University policy. D.S. is a paid consultant to iG and, D.S. and L.R.K. are paid members of iG's Scientific Advisory Board. The terms of this arrangement are being managed by the JHU in accordance with its conflict of interest policies.

\section{References}

[1] R. H. Taylor, S. Lavellee, G. C. Burdea, and R. Mosges, "Computer-integrated surgery. Technology and clinical applications. 1996," Clin Orthop, pp. 5-7, 1998.

[2] J. Yao, R. H. Taylor, R. P. Goldberg, R. Kumar, A. Bzostek, R. Van Vorhis, P. Kazanzides, and A. Gueziec, "A C-arm fluoroscopy-guided progressive cut refinement strategy using a surgical robot," Comput Aided Surg, vol. 5, pp. 373-90, 2000.

[3] R. H. Taylor, L. Joskowicz, B. Williamson, A. Gueziec, A. Kalvin, P. Kazanzides, R. Van Vorhis, J. Yao, R. Kumar, A. Bzostek, A. Sahay, M. Borner, and A. Lahmer, "Computer-integrated revision total hip replacement surgery: concept and preliminary results," Med Image Anal, vol. 3, pp. 301-19, 1999.

[4] N. Navab, B. Bascle, M. H. Loser, B. Geiger, and R. H. Taylor, "Visual Servoing for Automatic and Uncalibrated Needle Placement for Percutaenous Procedures," presented at IEEE Conference on Computer Vision and Pattern Recognition, 2000.

[5] A. Patriciu, D. Stoianovici, L. L. Whitcomb, T. Jarrett, D. Mazilu, A. Stanimir, I. Iordachita, J. Anderson, R. Taylor, and L. R. Kavoussi, "Motion-based robotic instrument targeting under C-Arm fluoroscopy," Medical Image Computing and Computer-Assisted Intervention - Miccai 2000, vol. 1935, pp. 988-998, 2000.

[6] D. Stoianovici, K. Cleary, A. Patriciu, D. Mazilu, A. Stanimir, N. Craciunoiu, V. Watson, and L. R. Kavoussi, "AcuBot: A Robot for Radiological Percutaneous Interventions," IEEE Transactions on Robotics and Automation, pp. to appear, 2002.

[7] S. Hutchinson, G. Hager, and P. Corke, "A Tutorial on Visual Servo Control," IEEE Transactions on Robotics and Automation, vol. 12, pp. 651-670, 1996.

[8] J. P. Hespanha, Z. Dodds, G. D. Hager, and A. S. Morse, "What tasks can be performed with an uncalibrated stereo vision system?," International Journal of Computer Vision, vol. 35, pp. 65-85, 1999.

[9] R. Kelly, "Robust Asymptotically Stable Visual Servoing of Planar Robots," IEEE Transactions on Robotics and Automation, vol. 12, pp. 759-766, 1996.

[10] Y. Shen, G. Xiang, Y.-H. Liu, and K. Li, "Uncalibrated Visual Servoing of Planar Robots," presented at IEEE International Conference on Robotics \& Automation, Washington, DC, 2002.

[11] D. Stoianovici, L. L. Whitcomb, J. H. Anderson, R. H. Taylor, and L. R. Kavoussi, "A modular surgical robotic system for image guided percutaneous procedures," Medical Image Computing and Computer-Assisted Intervention - Miccai'98, vol. 1496, pp. 404-410, 1998. 